\title{
Mannheim Partner Curves According to Darboux Frame in the Euclidean 3-space $E^{3}$
}

\author{
Abdullah Yıldırım* and Feryat Kaya
}

\begin{abstract}
In this study, we consider Mannheim partner curves according to Darboux Frame. Characterization of Mannheim partner curves lying on the surface are examined, and some curves are obtained. The characteristics of these curves are given by the geodesic curvatures, normal curvatures and geodesic torsions of these curves.
\end{abstract}

Keywords: Curves; Surfaces; Geodesics; Curvatures ; Frames .

AMS Subject Classification (2020): Primary: 53A04 ; Secondary: 53A05; 53C22; 53C21; 53 C99.

${ }^{*}$ Corresponding author

\section{Introduction}

The interest of special curves has increased recently. Some of these are associated curves. They are the curves one of the Frenet vectors at the corresponding points is equal to one of the Frenet vector of the other curve. One of the best examples of these curves is Mannheim partner curves. Mannheim curves are first defined by Mannheim in 1878 [6]. Mannheim partner curves are also defined by Liu and Wang [5].

Let $E^{3}$ be the 3-dimensional Euclidean space with the standard inner product and also two regular and unite speed curves be

$$
\begin{aligned}
& \alpha: I \subset R \quad \longrightarrow \quad E^{3} \\
& s \quad \longrightarrow \quad \alpha(s)=\left(\alpha_{1}(s), \alpha_{2}(s), \alpha_{3}(s)\right)
\end{aligned}
$$

and

$$
\begin{aligned}
& \alpha^{*}: \quad J \subset R \quad \longrightarrow \quad E^{3} \\
& s^{*} \quad \longrightarrow \alpha\left(s^{*}\right)=\left(\alpha_{1}^{*}(s), \alpha_{2}^{*}(s), \alpha_{3}^{*}(s)\right)
\end{aligned}
$$

in $E^{3}$. Here

$$
\begin{aligned}
h: I & \longrightarrow J \\
s & \longrightarrow h(s)=s^{*}
\end{aligned}
$$

is a homeomorphism function. If the principal normal vector field of the curve $\alpha$ and the binormal vector field of the curve $\alpha^{*}$ are linearly dependent, then these curves are called as Manheim partner curves. The pair $\left(\alpha, \alpha^{*}\right)$ is said to be a Mannheim pair.

It is just known that a space curve in $E^{3}$ is a Mannheim curve if and only if its curvature $\kappa$ and its torsion $\tau$ satisfy followings:

$$
\kappa=\lambda\left(\kappa^{2}+\tau^{2}\right) .
$$

Here $\lambda$ is nonzero constant [5]. Many researches have been conducted about Mannheim partner curves. Some of them Azak [7], Kazaz et al [3], Coşkun [1] and Kaymaz [2].

In this study, Mannheim partner curves are analyzed by using Darboux frame. We will research the states of geodesic curve, asymptotic curve and principle line of them.

Received : 01-08-2019, Accepted : 10-10-2020 


\section{Preliminaries}

Let the curve $\alpha: I \subset R \longmapsto E^{3}$ be a regular curve specified by arc parameter $s$. The triadic $\{T, N, B\}$ obtained as indicated below by means of this curve is called as Frenet vector field at the $\alpha(s)$ point of the curve $\alpha$.

$$
\begin{aligned}
& T(s)=\alpha^{\prime}(s), \\
& N(s)=\frac{\alpha^{\prime \prime}(s)}{\left\|\alpha^{\prime \prime}(s)\right\|}, \\
& B(s)=T(s) \times N(s) .
\end{aligned}
$$

In this point, $\times$ indicates vector product on $E^{3}$ and $\alpha^{\prime}(s)=\frac{\mathrm{d} \alpha}{\mathrm{d} s}$.

These vectors form an orthogonal vector system. In that point, $T$ is called as tangent vector field, $N$ and $B$ are called as principles normal vector field and binormal vector field, respectively. The Frenet formulas are given by as follows

$$
\left[\begin{array}{l}
T^{\prime} \\
N^{\prime} \\
B^{\prime}
\end{array}\right]=\left[\begin{array}{ccc}
0 & \kappa & 0 \\
-\kappa & 0 & \tau \\
0 & -\tau & 0
\end{array}\right]\left[\begin{array}{l}
T \\
N \\
B
\end{array}\right] .
$$

Here $\kappa$ and $\tau$ are called as curvature and torsion of the curve $\alpha$, respectively.

If the curve $\alpha$ is over a surface $S$, another frame which is called as Darboux frame and showed with $\{T, g, n\}$, could be found. In this frame, $T$ is tangent vector of the curve $\alpha, n$ is unit normal vector field of the surface $S$ and finally $g$ is another vector field written with $g=n \times T$. Relation between the Frenet frame and the Darboux frame is given by as follows

$$
\left[\begin{array}{l}
T \\
N \\
B
\end{array}\right]=\left[\begin{array}{ccc}
1 & 0 & 0 \\
0 & \cos \theta & -\sin \theta \\
0 & \sin \theta & \cos \theta
\end{array}\right]\left[\begin{array}{l}
T \\
g \\
n
\end{array}\right] .
$$

Here $\theta$ is the angle between $g$ and $N$. Derivative change of Darboux frames is as shown below:

$$
\left[\begin{array}{c}
T^{\prime} \\
g^{\prime} \\
n^{\prime}
\end{array}\right]=\left[\begin{array}{ccc}
0 & \kappa_{g} & \kappa_{n} \\
-\kappa_{g} & 0 & \tau_{g} \\
-\kappa_{n} & -\tau_{g} & 0
\end{array}\right]\left[\begin{array}{l}
T \\
g \\
n
\end{array}\right] .
$$

Here $\kappa_{g}, \kappa_{n}$ and $\tau_{g}$ in the equation above are called as geodesic curve, normal curve and geodesic torsion of the curve $\alpha$, respectively. The relationship between geodesic curvature, normal curvature, geodesic torsion and $\kappa, \tau$ are given in the figure below

$$
\kappa_{g}=\kappa \cos \theta, \quad \kappa_{n}=\kappa \sin \theta, \quad \tau_{g}=\tau+\theta^{\prime} .
$$

For the curve $\alpha$ in surface $S$, we say followings;

i. $\alpha$ is a geodesic curve if and only if $\kappa_{g}=0$,

ii. $\alpha$ is an asymptotic line if and only if $\kappa_{n}=0$,

iii. $\alpha$ is a principle curvature line if and only if $\tau_{g}=0$

[4].

In this study, we use $\{T, N, B, \kappa, \tau, s\}$ as Frenet elements of the curve $\alpha$ and $\left\{T^{*}, N^{*}, B^{*}, \kappa^{*}, \tau^{*}, s^{*}\right\}$ as Frenet elements of the curve $\alpha^{*}$.

Theorem 2.1. [5] Let $\left(\alpha, \alpha^{*}\right)$ be Mannheim partner curves. We write followings:

$$
\left[\begin{array}{l}
T \\
N \\
B
\end{array}\right]=\left[\begin{array}{ccc}
\cos \psi & \sin \psi & 0 \\
0 & 0 & 1 \\
-\sin \psi & \cos \psi & 0
\end{array}\right]\left[\begin{array}{l}
T^{*} \\
N^{*} \\
B^{*}
\end{array}\right] .
$$

Here, $\psi$ is angle between vectors $T$ and $T^{*}$.

- $\left(\tau^{*}\right)^{\prime}=\frac{\mathrm{d} \tau^{*}}{\mathrm{~d} s^{*}}=\frac{\kappa^{*}}{\lambda}\left(1+\lambda^{2}\left(\tau^{*}\right)^{2}\right)$. 


\section{Mannheim Partner Curves According to Darboux Frame in $E^{3}$}

In this part, we present the characterization of Mannheim partner curves by paying into consideration of Darboux frame.

Definition 3.1. Let $S$ and $S^{*}$ be directed two surface in $E^{3}$ and also the curves of $\alpha$ and $\alpha^{*}$ be given in the surfaces $S$ and $S^{*}$, respectively. If the curves $\alpha$ and $\alpha^{*}$ are Mannheim partner curves, these two curves are called as Mannheim partner curves in accordance with the surfaces $S$ and $S^{*}$. They are indicated as $\left(S_{\alpha}, S_{\alpha^{*}}^{*}\right)$.

We show the Darboux elements of the curve $\alpha$ as $\left\{T, g, n, \kappa_{g}, \kappa_{n}, \tau_{g}, s\right\}$ and the Darboux elements of the curve $\alpha^{*}$ as $\left\{T^{*}, g^{*}, n^{*}, \kappa_{g}^{*}, \kappa_{n}^{*}, \tau_{g}^{*}, s^{*}\right\}$ for Mannheim pair $\left(S_{\alpha}, S_{\alpha^{*}}^{*}\right)$. Here $\kappa_{g}, \kappa_{n}, \tau_{g}$ and $\kappa_{g}^{*}, \kappa_{n}^{*}, \tau_{g}^{*}$ are geodesic curvature, normal curvature, torsion curvature of the curves $\alpha$ and $\alpha^{*}, s$ and $s^{*}$ are arc elements of the curves $\alpha$ and $\alpha^{*}$, respectively.

Theorem 3.1. Let $\left(S_{\alpha}, S_{\alpha^{*}}^{*}\right)$ be Mannheim partner curves. Relation between Darboux frames are given as follows:

$$
\begin{aligned}
T= & (\cos \psi) T^{*}+\left(\sin \psi \cos \theta^{*}\right) g^{*}-\left(\sin \psi \sin \theta^{*}\right) n^{*}, \\
g= & -(\sin \theta \sin \psi) T^{*}+\left(\sin \theta^{*} \cos \theta+\sin \theta \cos \theta^{*} \cos \psi\right) g^{*} \\
& +\left(\cos \theta \cos \theta^{*}-\sin \theta^{*} \sin \theta \cos \psi\right) n^{*}, \\
n= & (\cos \theta \sin \psi) T^{*}+\left(-\sin \theta^{*} \sin \theta+\cos \theta^{*} \cos \theta \cos \psi\right) g^{*} \\
& -\left(\sin \theta \cos \theta^{*}+\sin \theta^{*} \cos \theta \cos \psi\right) n^{*},
\end{aligned}
$$

where, $\psi, \theta, \theta^{*}$ are the angles between $T$ and $T^{*}, g$ and $N, g^{*}$ and $N^{*}$, respectively.

Proof. From equation (2.1), the relation of the curve $\alpha$ between the Frenet frame and the Darboux frame is

$$
\left[\begin{array}{l}
T \\
N \\
B
\end{array}\right]=\left[\begin{array}{ccc}
1 & 0 & 0 \\
0 & \cos \theta & -\sin \theta \\
0 & \sin \theta & \cos \theta
\end{array}\right]\left[\begin{array}{l}
T \\
g \\
n
\end{array}\right]
$$

The relation of the curve $\alpha^{*}$ between the Frenet frame and the Darboux frame is

$$
\left[\begin{array}{l}
T^{*} \\
N^{*} \\
B^{*}
\end{array}\right]=\left[\begin{array}{ccc}
1 & 0 & 0 \\
0 & \cos \theta^{*} & -\sin \theta^{*} \\
0 & \sin \theta^{*} & \cos \theta^{*}
\end{array}\right]\left[\begin{array}{c}
T^{*} \\
g^{*} \\
n^{*}
\end{array}\right] .
$$

On the other side, the relation of the curves $\alpha$ and $\alpha^{*}$ between Frenet frames is

$$
\left[\begin{array}{l}
T \\
N \\
B
\end{array}\right]=\left[\begin{array}{ccc}
\cos \psi & 0 & \sin \psi \\
0 & 0 & 1 \\
-\sin \psi & 0 & \cos \psi
\end{array}\right]\left[\begin{array}{l}
T^{*} \\
N^{*} \\
B^{*}
\end{array}\right]
$$

Substituting the equation (3.4) in the equation (3.5), we obtain following equation:

$$
\left[\begin{array}{l}
T \\
N \\
B
\end{array}\right]=\left[\begin{array}{ccc}
\cos \psi & \sin \psi & 0 \\
0 & 0 & 1 \\
-\sin \psi & \cos \psi & 0
\end{array}\right]\left[\begin{array}{ccc}
1 & 0 & 0 \\
0 & \cos \theta^{*} & -\sin \theta^{*} \\
0 & \sin \theta^{*} & \cos \theta^{*}
\end{array}\right]\left[\begin{array}{c}
T^{*} \\
g^{*} \\
n^{*}
\end{array}\right] .
$$

Finally, by using (2.1) in (3.6), we finish the proof of the theorem.

Theorem 3.2. Let $\left(S_{\alpha}, S_{\alpha^{*}}^{*}\right)$ be Mannheim partner curves. We get following equations

$$
\begin{aligned}
& (\cos \psi) \frac{\mathrm{d} s^{*}}{\mathrm{~d} s}=1, \\
& (\sin \psi) \frac{\mathrm{d} s^{*}}{\mathrm{~d} s}=\lambda \tau^{*} .
\end{aligned}
$$


Proof. If $\left(S_{\alpha}, S_{\alpha^{*}}^{*}\right)$ is Mannheim partner curves, then we write

$$
\alpha(s)=\alpha^{*}(s)+\lambda B^{*}(s) .
$$

Taking the derivative of the equation (3.7) with respect to $s$ and use the Darboux frame, we find following equation

$$
T \frac{\mathrm{d} s}{\mathrm{~d} s^{*}}=T^{*}-\lambda \tau^{*} N^{*}+\lambda^{\prime} B^{*} .
$$

Multiplying both sides of the equation (3.8) by $B^{*}$, we obtain

$$
\lambda^{\prime}=0
$$

namely, $\lambda$ is constant. In this case, we get that the equation (3.8) is

$$
T \frac{\mathrm{d} s}{\mathrm{~d} s^{*}}=T^{*}-\lambda \tau^{*} N^{*}
$$

Again multiplying both sides of the equation (3.9) by $T^{*}$, we have

$$
(\cos \psi) \frac{\mathrm{d} s^{*}}{\mathrm{~d} s}=1,
$$

and multiplying both sides of the equation (3.9) by $N^{*}$, we obtain following

$$
(\sin \psi) \frac{\mathrm{d} s^{*}}{\mathrm{~d} s}=\lambda \tau .
$$

When we consider the Theorem 3.2, we give following corollary.

Corollary 3.1. Let Mannheim partner curves $\left(S_{\alpha}, S_{\alpha^{*}}^{*}\right)$ be specified. We have

$$
\tau_{g^{*}}=\frac{\left(\theta^{*}\right)^{\prime} \lambda-\tan \psi}{\lambda} .
$$

Proof. Considering Theorem 3.2, we have

$$
\frac{(\sin \psi) \frac{\mathrm{d} s^{*}}{\mathrm{~d} s}}{(\cos \psi) \frac{\mathrm{d} s^{*}}{\mathrm{~d} s}}=\lambda \tau^{*}=\tan \psi .
$$

We know that $\tau_{g}^{*}=\tau^{*}+\left(\theta^{\prime}\right)^{*}$. Hence, we finish the corollary.

Theorem 3.3. Let Mannheim partner curves $\left(S_{\alpha}, S_{\alpha^{*}}^{*}\right)$ be specified. We get following equation

$$
\kappa_{g} \sin \theta+\kappa_{n} \cos \theta=\left(\psi^{\prime}+\kappa_{g}^{*} \cos \theta^{*}-\kappa_{n}^{*} \sin \theta^{*}\right) \cos \psi
$$

Proof. From the equation (3.1), we oobtain

$$
T=(\cos \psi) T^{*}+\left(\sin \psi \cos \theta^{*}\right) g^{*}-\left(\sin \psi \sin \theta^{*}\right) n^{*} .
$$

Taking the derivative of the equation (3.1) with respect to $s$ and use the Darboux frame, we find following

$$
\begin{aligned}
\left(\kappa_{g} g+\kappa_{n} n\right) \frac{\mathrm{d} s}{\mathrm{~d} s^{*}}= & \left(-\psi^{\prime}-\kappa_{g}^{*} \cos \theta^{*}+\kappa_{n}^{*} \sin \theta^{*}\right) T^{*} \\
& +\left(\kappa_{g}^{*} \cos \psi-\psi^{\prime} \cos \psi \cos \theta^{*}-\left(\theta^{*}\right)^{\prime} \tau_{g} \sin \psi \sin \theta^{*}\right) g^{*} \\
& +\left(\begin{array}{l}
\kappa_{n}^{*} \cos \psi+\tau_{g}^{*} \sin \psi \cos \theta^{*}-\psi^{\prime} \cos \psi \sin \theta^{*} \\
-\left(\theta^{*}\right)^{\prime} \tau_{g} \sin \psi \cos \theta^{*}
\end{array}\right) n^{*} .
\end{aligned}
$$


Multiply both sides of the equation (3.11) by $T^{*}$, we obtain:

$$
\kappa_{g}<g, T^{*}>\frac{\mathrm{d} s}{\mathrm{~d} s^{*}}+\kappa_{n}<n, T^{*}>\frac{\mathrm{d} s}{\mathrm{~d} s^{*}}=\left(-\psi^{\prime}-\kappa_{g}^{*} \cos \theta^{*}+\kappa_{n}^{*} \sin \theta^{*}\right) \sin \psi .
$$

On the other side, multiplying both sides of the equations (3.2), and the equation (3.3) by $T^{*}$, we obtain following equations:

$$
\begin{aligned}
& <g, T^{*}>=-\sin \theta \sin \psi, \\
& <n, T^{*}>=\cos \theta \sin \psi .
\end{aligned}
$$

By using use these equations in the equation (3.12), we prove the theorem.

Considering Theorem 3.3, we find following corollaries.

Corollary 3.2. Let Mannheim partner curves $\left(S_{\alpha}, S_{\alpha^{*}}^{*}\right)$ be specified. Then we get following:

1. If both of the curves $\alpha$ and $\alpha^{*}$ are geodesic, then we have

$$
\kappa_{n}=\frac{\left(\psi^{\prime}-\kappa_{n}^{*} \sin \theta^{*}\right) \cos \psi}{\cos \theta}
$$

Here $\cos \theta \neq 0$.

2. If both of the curves $\alpha$ and $\alpha^{*}$ are asymptotic curves, then we get

$$
\kappa_{g}=\frac{\left(\psi^{\prime}+\kappa_{g}^{*} \cos \theta^{*}\right) \cos \psi}{\sin \theta} .
$$

Here $\sin \theta \neq 0$.

3. If $\alpha$ is an asymptotic curve and $\alpha^{*}$ is a geodesic curve, the we obtain

$$
\kappa_{g}=\frac{\left(\psi^{\prime}-\kappa_{n}^{*} \sin \theta^{*}\right) \cos \psi}{\sin \theta}
$$

Here $\sin \theta \neq 0$.

4. If $\alpha$ is a geodesic curve and $\alpha^{*}$ is an asymptotic curve, then we find

$$
\kappa_{n}=\frac{\left(\psi^{\prime}+\kappa_{g}^{*} \cos \theta^{*}\right) \cos \psi}{\cos \theta} .
$$

Here $\cos \theta \neq 0$.

\section{References}

[1] Coşkun, Ö.: 3-Boyutlu Öklid ve Minkowski Uzaylarında Mannheim Eğri Çiftleri. Ms. thesis. Uşak University (2010).

[2] Kaymaz, F., Kahraman, Aksoyak, F.: Some Special Curves and Mannheim Curves in Three Dimensional Euclidean Space. Mathematical Sciences and Applications E-Notes. 5(2), 34-39 (2017).

[3] Kazaz, M., Hüseyin, Uğurlu, H., Önder, M., Kahraman, T.: Manheim Partner D curves in the Euclidean 3-space $E^{3}$. New Trends in Mathematical Sciences. 3(2), 24-35 (2015).

[4] Kazaz, M., Hüseyin, Uğurlu, H., Önder, M., Oral, S.: Bertrand Partner D-Curves in the Euclidean 3-space $E^{3}$. Afyon Kocatepe Universty Journal of Science and Engineering. 16, 76-83 (2016).

[5] Liu, H., Wang, F.: Manheim Partner Curves in 3-Space. In: Proceeding of the Eleventh International Workshop on Differential Geometry, Daegu, SOUTH KOREA. 11, 25-31 (2007).

[6] Mannheim, A.: De l'emploi de la Courbe Représentative de la Surface des Normales Principales d'une Courbe Gauche Pour la Démonstration de Propriétés Relatives à Cette Courbure. Comptes Rendus des Séances de l'Académie des Sciences. 86, 1254-1256 (1878).

[7] Zeynep, Azak, A.: Üç Boyutlu Lorentz Uzayında Timelike Mannheim Ĕ̆ri Çifti Üzerine. Sakarya University Journal of Science and Art. 11(2), 35-45 (2009). 


\section{Affiliations}

ABDULLAH YILDIRIM

AdDRESS: Harran University, Science and Arts Faculty, Mathematics Department, 63300, Şanliurfa-Turkey. E-MAIL: abdullahyildirim@harran.edu.tr

ORCID ID:0000-0002-6579-3799

FERYAT KAYA

AdDrEsS: Şehit Abdülkadir Oğuz Anadolu İmam Hatip Lisesi, 63200, Şanlıurfa-Turkey.

E-MAIL: f.kaya73@outlook.com

ORCID ID:0000-0001-7725-0479 